\title{
Second Workshop on Teaching Computational Science WTCS 2008
}

\author{
A. Tirado-Ramos ${ }^{1}$ and Q. Luo ${ }^{2}$ \\ ${ }^{1}$ University of Amsterdam, Amsterdam, The Netherlands \\ ${ }^{2}$ Wuhan University of Science and Technology, Zhongnan, China \\ alfredo@science.uva.nl, ccnu_luo2008@yahoo.com.cn
}

\begin{abstract}
The Second Workshop on Teaching Computational Science, within the International Conference on Computational Science, provides a platform for discussing innovations in teaching computational sciences at all levels and contexts of higher education. This editorial provides an introduction to the work presented during the sessions.
\end{abstract}

Keywords: computational science, teaching, parallel computing, e-Learning, collaborative environments, higher education.

\section{Introduction}

Experience shows that students who have been trained in technology-based environments, such as computational science, tend to thrive in today's technology-driven societies. The interdisciplinary nature of computational science allows the integration of methods from computer science, mathematical modeling, and data visualization, among others, in order to create virtual laboratories for in-silico experimentation that just a few years ago would have proved costly and impractical for most academic institutions of higher learning. It is evident that the interaction of computational methods allows more intriguing questions to be posed by teachers and students at lower cost experimental settings [1].

The field of higher education is therefore currently witnessing the rapid adoption of computational tools and methods by science teachers. A large majority of those teachers have steadily joined forces and shared experiences in the last few years on the use of high performance computing facilities in order to promote the benefits and importance of computational science instruction in science classrooms.

The International workshop on Teaching Computational Science (WTCS2008), held in Krakow, Poland, in conjunction with the International Conference on Computational Science 2008 (ICCS 2008) offers a technical program consisting of presentations dealing with the state of the art in the field. The workshop includes presentations that describe innovations in the context of formal courses involving, for example, introductory programming, service courses and specialist undergraduate or postgraduate topics.

During the workshop sessions, Gimenez et al present their experiences with the use of metaheuristics in a parallel computing course, mapping problems in which processes are assigned to processors in a heterogeneous environment, with heterogeneity 
in computation and in the network. Freitag et al discuss how to introduce students to collaborative work, in project-based learning courses on computer network applications. Hamada et al provide their experiences with e-Learning using supporting active tools to improve learning and evaluation. Hnatkowska et al present an assessment approach to software development process used within student team projects, based on the Process Assessment Model. Aracely et al present their experiences teaching cryptography to engineering students, using Maple software in a graduate-level course. Iglesias et al discuss teaching in the context of the European space of higher education, focusing on the problem of teaching computer graphics. Ramos-Quintana et al elaborate on collaborative environments to encourage self-directed learning, focusing on their experiences with object-oriented programming and case-based reasoning. Shiflet et al discuss theirs undergraduate computational science curriculum, which served as a basis the first textbook designed specifically for an introductory course in the computational science and engineering curriculum. Finally, GonzalezCinca et al discuss innovative ways of teaching computational science in aeronautics, with a particular emphasis in Computational Fluid Dynamics, and the experiences derived from implementation.

We feel that the width and breadth of topics shown at the workshop provide a glimpse on the current state of the art in the field, and a promising window to challenges and possibilities ahead.

Acknowledgments. The workshop chairs would like to thank all the work by the scientific reviewing committee, as well as the efforts by ICCS chairs Dick van Albada, Marian Bubak, and Peter Sloot.

\section{Reference}

1. Selwyn, N.: The use of computer technology in university teaching and learning: a critical perspective. Journal of Computer Assisted Learning 23(2), 83-94 (2007) 